\title{
Spontaneous Height Restoration of Vertebral Compression Fracture - A Case Report-
}

Department of Anesthesiology and Pain Medicine, Seoul National University Hospital, Seoul, *Seoul National University Bundang Hospital, Seongnam, Korea

Young Joo, MD, Pyung Bok Lee, MD*, and Francis Sahngun Nahm, MD*

Vertebral compression fractures result in vertebral height loss and alter sagittal spinal alignment, which in turn can lead to increased morbidity and mortality. Acute osteoporotic vertebral compression fractures are known to increase mobility and instability of the spine. There are limited published data correlating the degree of dynamic mobility and the efficacy of kyphoplasty on vertebral compression fractures. Here we report a 73-year-old female with a severe acute osteoporotic L2 compression fracture who obtained total vertebral height restoration following kyphoplasty, with resolution of back pain. (Korean J Pain 2011; 24: 235-238)

Key Words:

compression fracture, dynamic mobility, kyphoplasty, osteoporosis, spine.

Vertebral compression fractures (VCFs) are primarily caused by osteoporosis. As the population ages, the incidence of VCFs is also likely to increase [1]. The clinical characteristics of VCFs are loss of vertebral height and acute pain. Radiographic images are the standardized protocol to diagnose VCFs, with changes in vertebral height revealed on AP and lateral views [2]. However, due to the increased dynamic mobility (DM) of vertebrae at the site of a fracture, transient spontaneous restoration of vertebral height is also common. Therefore, depending on the patient's posture, acute compression fracture or loss of height may not be visualized on a static image, possibly leading to inaccurate diagnosis and treatment. Dynamic mobility of vertebral compression fractures can be measured by comparing standing and supine images. However, there is limited information available in the literature on the extent and frequency of this phenomenon. We describe a case of acute vertebral compression fracture with a high degree of dynamic mobility and pain treated with kyphoplasty.

\section{CASE REPORT}

A seventy-three-year-old woman with a 2-week his-

Received August 9, 2011. Revised October 5, 2011. Accepted October 6, 2011.

Correspondence to: Francis Sahngun Nahm, MD

Department of Anesthesiology and Pain Medicine, Seoul National University Bundang Hospital, 166, Gumi-ro, Bundang-gu, Seongnam 463-707, Korea

Tel: +82-31-787-7499, Fax: +82-31-787-4063, E-mail: hiitsme@snubh.org

(a) This is an open-access article distributed under the terms of the Creative Commons Attribution Non-Commercial License (http:// creativecommons.org/licenses/by-nc/3.0/), which permits unrestricted non-commercial use, distribution, and reproduction in any medium, provided the original work is properly cited.

Copyright (c) The Korean Pain Society, 2011 
tory of severe back pain after a fall was referred to our pain clinic. Bed rest and conservative therapies had failed to provide relief, and the patient rated her pain level as 9 out of $10 \mathrm{~cm}$ on the visual analogue scale (VAS). She had a history of osteoporosis and had been taking bisphosphonates for the past 1 year.

The initial lumbar magnetic resonance imaging (MRI) study revealed marrow edema at the L2 vertebra, but no definite compression fracture (Fig. 1). Due to the severity

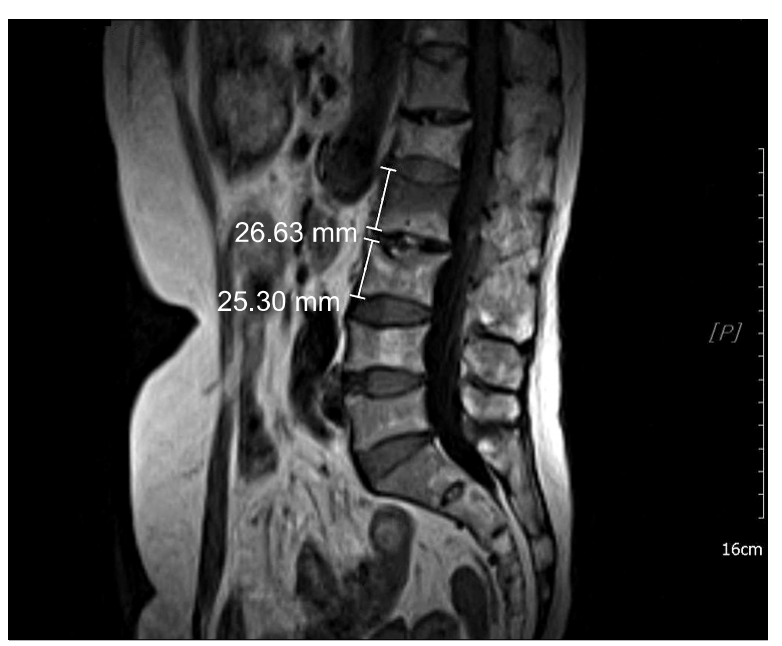

Fig. 1. T1 weighted image of lumbar MRI. There is no height loss except bone marrow edema in $2^{\text {nd }}$ lumbar vertebra.

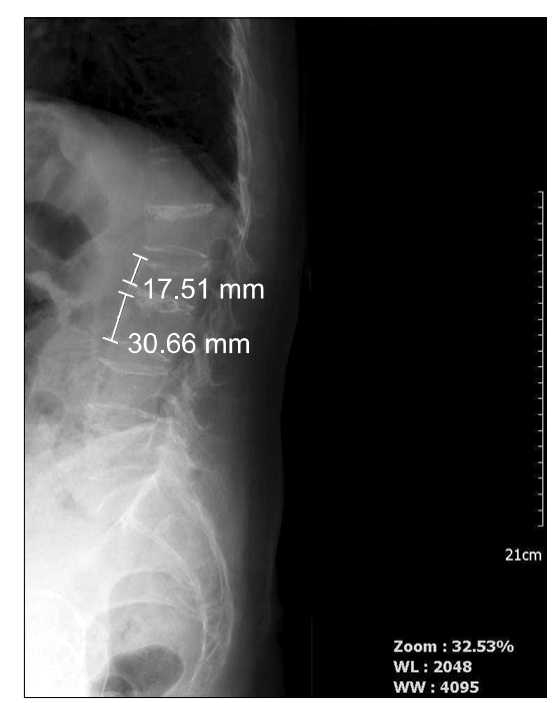

Fig. 2. Preoperative standing anterior-posterior radiographic study showing the anterior height of L2 vertebral was $56.7 \%$ compared to that of L3. of the patient's pain, we performed a fluoroscopicallyguided lumbar epidural injection with a mixture of triamcinolone $20 \mathrm{mg}$ and $0.18 \%$ levobupivacaine $8 \mathrm{ml}$. She was discharged home on Ultracet ${ }^{\circledR}$ and nortriptyline. However, three days later the patient returned to the pain clinic with progressively worsening back pain. On a repeat lumbar MRI, an acute vertebral compression fracture was noted at L2. The anterior height of the L2 vertebral body was only $54.80 \%$ of that of L3 (Fig. 2). Subsequently, the decision was made to proceed with kyphoplasty of the L2 vertebral body.

The procedure consisted of placing the patient in the prone position, with bolsters at the upper thorax and pelvis to allow extension of the spine. The lateral fluoroscopy view confirmed the total height restoration of the L2 vertebral body (Fig. 3). After sterile preparation of the skin, kyphoplasty was performed bilaterally under fluoroscopic guidance.

After the drill was advanced through the access cannula, the balloon bone tamp was placed in the cavity. The cement mixture (polymethyl methacrylate, barium sulfate) was injected slowly, ensuring no cement leaks from the borders of the created bone void (Fig. 4). The wounds were cleaned and dressed, and the patient was monitored overnight prior to discharge home in satisfactory condition. There were no complications from the procedure, and the patient reported a VAS score of 2 out of 10 at the time of discharge.

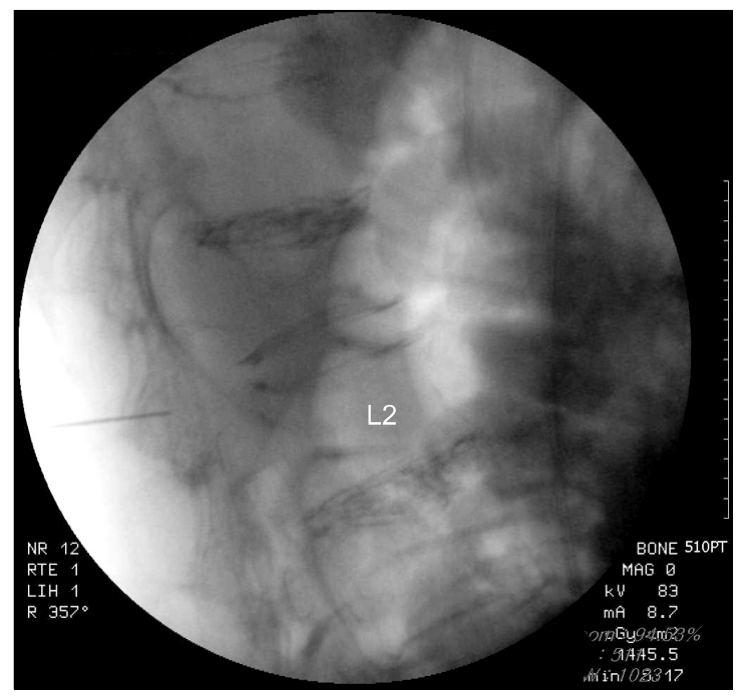

Fig. 3. Prone cross-table lateral fluoroscopy was performed and we found the height restoration of L2 vertebral body. 

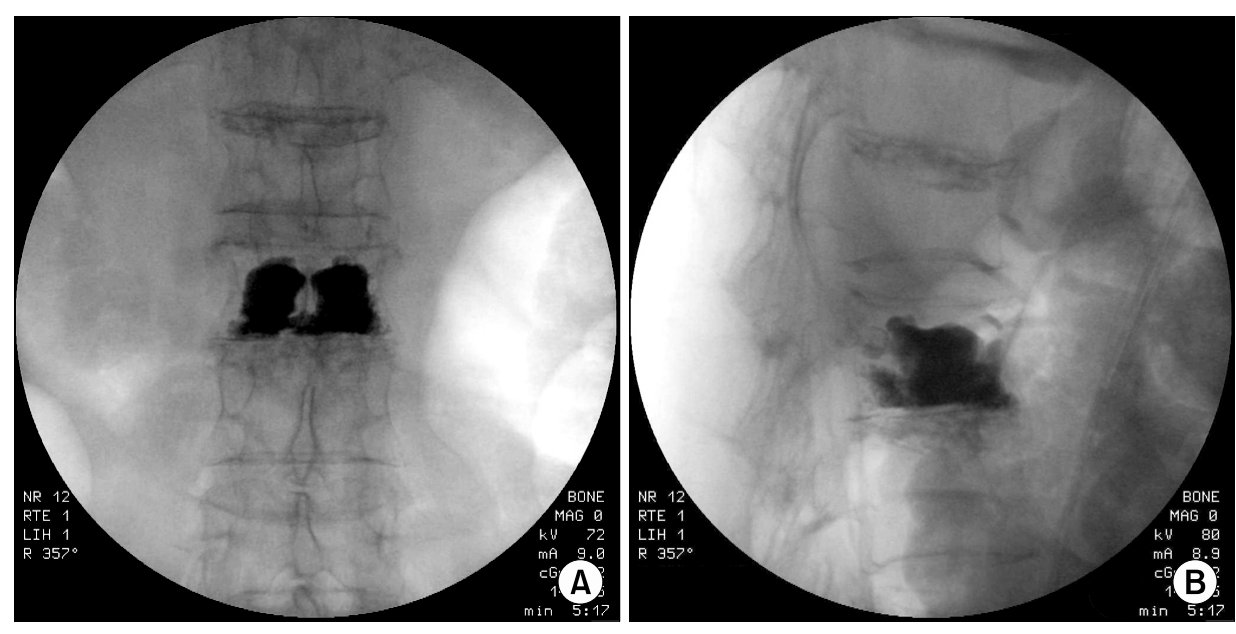

Fig. 4. After bone cement injection. Anterior-posterior view (A), and lateral view (B).

\section{DISCUSSION}

This case demonstrated that total height restoration of a vertebral body can occur spontaneously due to dynamic mobility of the fractured vertebra. McKiernan et al. [2] defined dynamic mobility as "any measurable change in vertebral body height occurred between standing and supine radiograph," and this phenomenon could be found in $44 \%$ of VCF patients. The reported degree of dynamic mobility ranged from $48 \%$ to $80 \%$ of that of normal adjacent vertebrae. It has been demonstrated that the apparent magnitude of height restoration varied nearly fourfold depending on the initial fracture severity and the measuring method on radiograph [3]. Many VCFs are mobile, and mere positional adjustment can lead to clinically significant height restoration [3]. The diagnosis of acute VCF can therefore be elusive due to the dynamic mobility of the fracture site. A fracture may not be visible on initial radiographs, but may become evident on radiographs two or three weeks later as the osteoporotic bone settles [4]. Similarly, our initial image study also showed minimal height loss, with progression of vertebral height loss visible in the follow-up radiographic images.

Vertebral height restoration is important because the greater the deformity, the greater the likelihood of pain and disability [5]. The spontaneously restored vertebra can be identified by the presence of air [2,3]. This spontaneous re-establishment of vertebral height is transient and structurally unstable. Such findings can give a false sense of structural integrity, leading to inappropriate symptom management. In our case, the height restoration was achieved spontaneously, and the patient continued to complain of pain, likely due to the vertebral instability.

Shindle et al. [1] indicated that vertebral height restoration without bone cement injection did not prove to provide any benefits to patients. VCFs can be restored by position, but kyphoplasty is superior to the positioning maneuver alone [1,2]. VCF restoration due to dynamic mobility can be obtained on imaging studies. However, VCF restoration either by position or air is not sufficient to achieve appropriate symptom resolution, and kyphoplasty should be perfomed. Kyphoplasty is known to be an effective treatment method for the osteoporotic compression fracture [6].

Shindle and coworkers demonstrated that the positioning maneuver provided an average of $10.4 \%$ height restoration, while kyphoplasty provided an average of $57.0 \%$ restoration in the central portion of the vertebra [1]. Kyphoplasty may be either unsafe or technically impossible in the presence of severe vertebral body height loss (vertebra plana). However, assessment of dynamic mobility is recommended on all acute vertebral fractures via serial imaging studies. Outcome studies on the basis of the initial radiographic appearance of vertebral fractures have shown that patients who have more severe pain initially, and a well-defined wedge fracture, may do well with acute pain management and early mobilization [7]. Although there is little correlation between the degree of collapse of the vertebral body and the level of pain, vertebral height restoration is essential to minimize pain and disability. Therefore, kyphoplasty is recommended to restore vertebral height in VCFs, especially when a high degree of dynamic mobility is present. 


\section{REFERENCES}

1. Shindle MK, Gardner MJ, Koob J, Bukata S, Cabin JA, Lane JM. Vertebral height restoration in osteoporotic compression fractures: kyphoplasty balloon tamp is superior to postural correction alone. Osteoporos Int 2006; 17: 1815-9.

2. McKiernan F, Jensen R, Faciszewski T. The dynamic mobility of vertebral compression fractures. J Bone Miner Res 2003; 18: 24-9.

3. Mckiernan F, Faciszewski T, Jensen R. Reporting height restoration in vertebral compression fractures. Spine (Phila Pa 1976) 2003; 28: 2517-21.
4. Rao RD, Singrakhia MD. Painful osteoporotic vertebral fracture. Pathogenesis, evaluation, and roles of vertebroplasty and kyphoplasty in its management. J Bone Joint Surg Am 2003; 85-A: 2010-22.

5. Silverman SL. The clinical consequences of vertebral compression fracture. Bone 1992; 13 Suppl 2: S27-31.

6. Han KR, Kim C, Yang JY, Han ST, Kim YS. Balloon kyphoplasty for the treatment of vertebral compression fractures. Korean J Pain 2006; 19: 56-62.

7. Lyritis GP, Mayasis B, Tsakalakos N, Lambropoulos A, Gazi $\mathrm{S}$, Karachalios T, et al. The natural history of the osteoporotic vertebral fracture. Clin Rheumatol 1989; 8 Suppl 2: 66-9. 